\title{
Titanium Implant Devices: Increase Biocompatibility or Decrease Drug Release Rate?
}

\author{
Mariana de Souza Sikora* \\ Department of Chemistry, Universidade Tecnológica Federal do Paraná (UTFPR), Brazil \\ *Corresponding author: Mariana de Souza Sikora, Department of Chemistry, Universidade Tecnológica Federal do Paraná (UTFPR), \\ Campus Pato Branco, Via do Conhecimento Km 1, 85503-390, Pato Branco, Brazil.
}

To Cite This Article: Mariana de Souza Sikora, Titanium Implant Devices: Increase Biocompatibility or Decrease Drug Release Rate?. Am J Biomed Sci \& Res. 2020 - 7(1). AJBSR.MS.ID.001106. DOI: 10.34297/AJBSR.2020.07.001106.

Received: 眥 September 24, 2019; Published: 眥 January 22, 2020

\section{Mini-Review}

Titanium and its alloys are the most used metallic materials for the manufacture of orthopedic and dental prostheses due to their similarities to human bone. Among these properties, the hardness and Young's modulus stand out, besides being chemically inert and presenting low corrosion potential in biological environments [1]. Its low corrosion rate is due to the formation of a $\mathrm{TiO}_{2}$ layer, also known as a "barrier oxide" responsible for metal passivation.

$\mathrm{TiO}_{2}$ formation is a thermodynamically favorable process, and an oxide of a few nanometer thicknesses is formed as soon as the metal is exposed to an oxygen-containing environment. Due to this factor, the characteristics of the coating can be easily experimentally manipulated. One of the techniques that have been widely used for implant surface modification is anodization. Through this technique, it is possible to create from compact films, as pores of the order of micrometers, or even nanotubes that can have diameters ranging from $30-100 \mathrm{~nm} \mathrm{[2,3].}$

Over the course of several years, much effort has been put into the development of these structures as well as their possible applications. It is now possible to obtain highly organized nanostructures of $100-1000 \mu \mathrm{m}$ in length, which can undoubtedly have many advantages for applications requiring materials with a high surface area. For implantable materials, nanoarchitecture provides a large area for osteoblast growth and cell differentiation, which favors the osseointegration process. With all these characteristics, it was not difficult to think about the possibility of using implants with nanostructured surfaces to produce drug delivery devices.

The main problem with the placement of orthopedic and dental implants is the rejection of the material by the human body. The process of implant rejection usually occurs by contamination of the material with microorganisms, with Staphylococcus Aureus being the most common, which grows on the implant forming a bacterial biofilm. After the formation of this biofilm, it is practically impossible to recover the surface of the biomaterial. Thus, an idea to reduce the incidence of this problem could be the incorporation of antimicrobial drugs. Such devices would act locally, reducing the amount of intravenous or oral drugs prescribed to patients postoperatively.

Several studies have proven the potential of this material to produce this device. Two properties are desirable in these materials, the first one is the bioactivity, i.e., the ability of the implant to Osseo integrate. Such ability is observed in materials with high wettability, which facilitates adhesion of osteoblastic cells and facilitates the osseointegration process. The other important feature is a slow drug release rate so that the drug concentration in the bloodstream remains within the range required for inhibition of the microorganisms. The problem with this latter feature is that the antimicrobial drugs used are water-soluble, so the wettability of the device is once again of great importance in the development of the material, but in an antagonistic manner.

The lower the wettability, the lower the drug release rate. Many works show the addition of diffusional barriers to the implant surface to decrease the drug release rate $[4,5]$. However, the great effort made to obtain a large surface area is lost in this case. Although coatings with smaller nanotubes have larger surface areas, the effect of capillarity on these materials is more pronounced and cannot be neglected in the development of biomaterials of this kind [5]. Therefore, further investigation of the ideal conditions for a high osseointegration rate coupled with a low drug release rate is needed. In recent studies we observed that nanotubular films incorporated with ciprofloxacin can release $1 \mathrm{mg}$ of the drug in $8 \mathrm{~min}$ or $300 \mathrm{~min}$, depending on the experimental conditions of the biomaterial synthesis [5]. However, the question that remains is what would be the ideal condition? A lot of research is still needed to answer this question. 


\section{References}

1. Saha R, Nix WD (2002) Effects of the substrate on the determination of thin film mechanical properties by nanoindentation. Acta Mater 50(1): 23-38.

2. Mansoorianfar M, Tavoosi M, Mozafarinia R, Ghasemia A Doostmohammadi A (2017) Preparation and characterization of TiO2 nanotube arrays on Ti6Al4V surface for enhancement of cell treatment. Surf Coatings Technol 321: 409-415.

3. Kulkarni M, Mazare A, Gongadze E, Perutkova Š, Kralj-Iglič V, et al. (2015) Titanium nanostructures for biomedical applications. Nanotechnology 26(6): 18.
4. Kumeria T, Mon H, Aw MS, Gulati K, Santos A, et al. (2015) Advanced biopolymer-coated drug-releasing Titania Nanotubes (TNTs) implants with simultaneously enhanced osteoblast adhesion and antibacterial properties. Colloids Surfaces B Biointerfaces 130: 255-263.

5. CH Ferreira, AP Simon, VAQ Santos, Santos JS, Trivinho-Strixino F, et al. (2019) Nanotexturization of Ti-based implants in simulated body fluid: Influence of synthesis parameters on coating properties and kinetics of drug release. J Mater Res 34(16): 2828-2836. 